\title{
MEASURES ORTHOGONAL TO ALGEBRAS AND SETS OF ANTISYMMETRY
}

\author{
IRVING GLICKSBERG(1)
}

1. We shall be concerned with two items concerning function algebras, connected in large part by their relation to the measures orthogonal to a given algebra: the expression of a general function algebra in terms of antisymmetric algebras recently obtained by Bishop [4], and the question of interpolation in such algebras.

Let $X$ be a compact Hausdorff space, $C(X)$ the usual algebra of continuous complex functions on $X$, and $A$ a closed subalgebra of $C(X)$ containing the constants. Silov [16] called $A$ antisymmetric if every real valued element of $A$ is constant, and inquired whether (as a result of his [13, p. 127] partially suggested) every algebra of continuous functions could be expressed in terms of antisymmetric algebras. Recently Bishop [4] obtained the desired expression, following an approach suggested by Silov's work. In the first part of this paper we shall give a simpler approach to Bishop's theorem and some consequences.

Call subset $K$ of $X$ a set of antisymmetry (or an antisymmetric set) of $A$ if, for $f$ in $A, f$ real valued on $K$ implies $f$ is constant on $K$. Let $f \mid K$ be the restriction of $f$ to $K, A \mid K=\{f \mid K: f \in A\}$. Then we can restate Bishop's theorem as

THEOREM 1.1. Every antisymmetric set of $A$ is contained in a maximal antisymmetric set. The collection $\mathscr{K}$ of maximal antisymmetric sets forms a pairwise disjoint, closed covering of $X$ satisfying

(a) $f \in C(X)$ and $f|K \in A| K$ for every $K$ in $\mathscr{K}$ imply $f \in A$;

(b) $A \mid K$ is closed in $C(K), K \in \mathscr{K}$.

The only nontrivial assertions are (a) and (b), of which the first generalizes the Stone-Weierstrass theorem. (When $A$ is conjugate closed and separates the points of $X$, so that antisymmetric sets reduce to points, (a) is precisely StoneWeierstrass.) Our proof of (a) is based on consideration of measures orthogonal to $A$ and application of the Krein-Milman theorem, and is simply a modification of deBranges' proof of the Stone-Weierstrass theorem [6].

In succeeding sections we consider some interpolation questions and their relation to measures orthogonal to $A$, as well as their relation to $\mathscr{K}$. In $\$ 4$ we generalize a result of Rudin [14] on norm preserving interpolation by continuous analytic functions on the disc to dirichlet algebras.

Received by the editors October 26, 1961.

(1) Supported in part by National Science Foundation through Grant G14779, and in part by the Air Force Office of Scientific Research. 
Our results were stimulated by, and are based upon, the results of several authors (in particular deBranges [6] and Bishop $[2 ; 3 ; 4 ; 5]$ ) and also conversations with various friends, especially $\mathrm{K}$. deLeeuw, to whom the author would like to express his thanks.

1.2. Notation. $M(X)$ will denote the usual Banach space of all complex finite regular Borel measures on $X$, the dual of the space $C(X)$ of continuous functions; $M^{R}(X)$ and $C^{R}(X)$ will be used to represent the real valued elements of these spaces. For $\mu$ in $M(X)$ we shall employ the notational abuse: $\mu(f)=\int f d \mu$. For any function $f, f \mu$ will denote the usual product of function and measure; as a functional on $C(X), f \mu(g)=\mu(f g)=\int f g d \mu, g$ in $C(X)$. $|\mu|$ will denote the unique non-negative element of $M(X)$ with $\mu=f|\mu|$, where $f$ is a unimodular Borel function, and carrier $\mu$ will denote the complement of all open sets of $|\mu|$ measure zero. $\mu_{K}$ will denote the restriction of $\mu$ to $K \subset X: \mu_{K}(E)=\mu(K \cap E)$.

We use $\perp$ to denote orthogonality between elements of $C(X)$ and $M(X)$; for example $\mu \perp A$ means $\mu(f)=0, f \in A$, and $A^{\perp}$ will denote the subspace of $M(X)$ formed by such $\mu$. And ball $A^{+}$will denote the elements of norm $\leqq 1$ of this subspace, (ball $\left.A^{+}\right)^{e}$ the set of extreme points of this set.

$\operatorname{Re} f$ will denote the real part of $f ; \operatorname{Re} A$ will similarly denote $\{\operatorname{Re} f: f \in A\}$.

2. Proof of Bishop's theorem. We begin with some obvious remarks on antisymmetric sets. Since the union of all antisymmetric sets containing a given one is clearly antisymmetric, and just as clearly maximal, each antisymmetric set is contained in a maximal antisymmetric set. In particular this applies to single element sets (clearly antisymmetric!), so $\mathscr{K}$ covers $X$; moreover the antisymmetry of $K$ clearly implies that of $K^{-}$since $A \subset C(X)$, so each $K$ in $\mathscr{K}$ is closed. And trivially the union of two antisymmetric sets which meet is again antisymmetric, so the elements of $\mathscr{K}$ are pairwise disjoint; thus the initial statements of 1.1 are established.

Next we have (cf. deBranges [6])

LemMa 2.1. Let $\mu \in\left(\text { ball } A^{+}\right)^{e}$. Then carrier $\mu$ is a set of antisymmetry.

Proof. Suppose $f$ in $A$ is real valued on $K=$ carrier $\mu$; we shall show $f$ is constant on $K$. Since $A$ contains the constants we can clearly assume $0<f<1$ on $K$.

Now $f \mu$ and $(1-f) \mu$ are nonzero measures, so we may write

$$
\mu=\|f \mu\| \frac{f \mu}{\|f \mu\|}+\|(1-f) \mu\| \frac{(1-f) \mu}{\|(1-f) \mu\|}
$$

while

$$
\begin{aligned}
\|f \mu\|+\|(1-f) \mu\| & =\int|f| d|\mu|+\int|1-f| d|\mu| \\
& =\int f d|\mu|+\int(1-f) d|\mu|=\int 1 d|\mu|=1
\end{aligned}
$$


But $f \mu$ and $(1-f) \mu$ lie in $A^{+}$since $f \mu(g)=\mu(f g)=0$ for $g$ in $A$. So (2.1), (2.2) and the extremity of $\mu$ imply $\mu=f \mu /\|f \mu\|$, whence $f$ is constant on $K=$ carrier $\mu$ as desired.

To complete our proof of (a) note that our $f$ in $C(X)$, if not in $A$, cannot be orthogonal to $A^{+}$by the Hahn-Banach theorem, or, equivalently, cannot be othogonal to ball $A^{+}$. From the Krein-Milman theorem we conclude that $f$ is not orthogonal to some $\mu$ in (ball $\left.A^{+}\right)^{e}$. But by 2.1 carrier $\mu$ is a set of antisymmetry, and so is contained in some $K$ in $\mathscr{K}$, on which $f=g$ for some $g$ in $A$ by hypothesis. Thus $\mu(f)=\int f d \mu=\int g d \mu=0$, the desired contradiction, yielding (a).

To obtain 1.1 (b) we require some terminology. Call $E \subset X$ a peak set (of $A$ ) if $E=\{x: f(x)=1\}$, where $f \in A$ has $\|f\|=1$; replacing $f$ by $(1+f) / 2$, we can assume $|f(x)|<1$ for $x \notin E$, and shall then say $f$ peaks on $E$. The following lemma appears in $[1 ; 10]$; for convenience we repeat its proof.

LEMMA 2.2. If $E$ is an intersection of peak sets, $A \mid E$ is closed in $C(E)$; in fact $A \mid E$ is isometrically isomorphic to the quotient algebra $A / k E$, where $k E=\{f: f \in A, f(E)=0\}$.

Here as usual, the norm in the quotient is defined as

$$
\|f+k E\|=\inf \{\mid f+g \|: g \in k E\},
$$

while $\|f \mid E\|=\sup \{|f(x)|: x \in E\}$ will denote the norm of $f|E \in A| E$. Since multiplicative linear functionals on $A / k E$ are of norm $\leqq 1,|f(x)| \leqq\|f+k E\|$, $x \in E$, so $\|f \mid E\| \leqq\left\|f^{\prime}+k E\right\|$. If $V$ is an open neighborhood of $E$ on which $|f| \leqq\|f \mid E\|+\varepsilon$ then some finite intersection $F$ of the peak sets containing $E$ is contained in $V$ by compactness; trivially $F$ is again a peak set, and so there is a $g$ in $A$ with $g(F)=1$, and $|g(x)|<1$ for $x \notin F$. But $g^{n} f \in f+k E$ and lim sup $\left\|g^{n} f\right\|$ $\leqq \sup |f(V)| \leqq\|f \mid E\|+\varepsilon ;$ consequently $\|f+k E\| \leqq\|f \mid E\|$ and $\|f+k E\|$ $=\|f \mid E\|$. Thus $A \mid E$, in the norm of $C(E)$, is isometrically isomorphic with the complete algebra $A / k E$, and so closed.

LEMMA 2.3. Every maximal antisymmetric set $K$ is an intersection of peak sets.

Note that every (nonempty) countable intersection of peak sets is a peak set (if $f_{n}$ peaks on $E_{n}, \Sigma 2^{-n} f_{n}$ peaks on $\bigcap E_{n}$ ). $X$ is of course a peak set containing $K$; let $E$ denote the intersection of all peak sets containing $K$.

Suppose $E \neq K$. Since $K \subset E, E$ is not a set of antisymmetry so there is an $f$ in $A$ with $f \mid E$ nonconstant and real valued. Now the compact set $f(E)$ is a $G_{\delta}$ in the complex plane, so $f^{-1}(f(E))$ is a $G_{\delta}$ in $X: f^{-1}(f(E))=\bigcap V_{n}, V_{n}$ open. But by compactness each neighborhood $V_{n}$ of $E$ contains a finite intersection $F_{n}$ of peak sets, and thus $E \subset F=\bigcap F_{n} \subset \bigcap V_{n}=f^{-1}(f(E))$, where $F$ is a peak set, and $f(F)=f(E)$.

Consequently $f \mid F$ is a nonconstant real function while $f$ is constant on $K$, 
so clearly there is a polynomial $p$ for which $p(f) \mid F$ peaks on a proper subset $L$ of $F$ containing $K$. Now by an argument of Bishop [3, last paragraph of p. 631] or by $[13,3.3 .13$, p. 140] if $F$ is a peak set of $A$ and $L \subset F$ is a peak set of $A \mid F$ then $L$ is a peak set of $A$, so our $L$ is a peak set of $A$. But $p(f(E))=p(f(F))$ shows $L$ cannot contain $E$, as all peak sets containing $K$ must. Thus we have arrived at the desired contradiction, showing $E \neq K$ is false, and completing the proof of 2.3 .

Combining 2.2 and 2.3 we obtain (b), and in fact more.

Corollary 2.4. For $K$ in $\mathscr{K}, A \mid K$ is isometrically isomorphic to $A / k K$.

As we shall see later (cf. 4.5) this (and 2.2) can be strengthened by an application of the argument of Bishop cited above.

It should be noted that the decomposition space produced by $\mathscr{K}$ is not, in general, Hausdorff. This is shown by an example used by Silov to show his result [16] did not express $A$ in terms of antisymmetric algebras; an analogous example is given in $\$ 5$.

We turn next to some properties of $\mathscr{K}$ which follow (essentially) from the argument used to obtain 1.1. First note the following property of $\mathscr{K}$ : if $f$ in $C^{R}(X)$ has $f \mid K \in(\operatorname{Re} A \mid K)^{-}$(uniform closure in $C^{R}(K)$ ) for every $K$ in $\mathscr{K}$, then $f \in(\operatorname{Re} A)^{-}$(closure in $\left.C^{R}(X)\right)$. Indeed if the conclusion fails there is a real measure $\mu$ with $\mu(f) \neq 0$ and $\mu \perp \operatorname{Re} A$ (which, for real measures, amounts precisely to $\mu \perp A)$; let $\mu$ in fact be chosen so as to be extreme in $\left\{v: v \in M^{R}(X), v \perp A,\|v\|\right.$ $\leqq 1\}$. Then if

$$
\mu=a v_{1}+(1-a) v_{2}, \quad 0<a<1, \quad v_{i} \in \text { ball } A^{+}, \quad i=1,2,
$$

it is a simple matter to conclude that each $v_{i}$ is real, and so coincides with $\mu$. Consequently 2.1 applies to $\mu$, and as before we obtain a contradiction.

Next we have the following result, which relates the ideal structure of $A$ to $\mathscr{K}$.

THEOREM 2.5. Let I be a closed ideal in A. Then

(a') $f \in C(X)$ and $f|K \in I| K$ for every $K$ in $\mathscr{K}$ imply $f \in I$,

(b') $I \mid K$ is closed in $C(K)$ (or in $A \mid K), K \in \mathscr{K}$.

Thus every closed ideal $I$ of $A$ produces closed ideals $I \mid K$ from which $I$ can be recovered. ( $\left.\mathrm{a}^{\prime}\right)$ follows exactly as $1.1(\mathrm{a})$, once it is noted that $\mu \in\left(\text { ball } I^{+}\right)^{e}$ implies carrier $\mu$ is a set of antisymmetry of $A$, and this follows from the proof of 2.1 as soon as we note that $f \in A$ and $\mu \perp I$ imply $f \mu \perp I$.

The proof of $\left(b^{\prime}\right)$ can be obtained as before. For we have a natural isomorphism of the Banach algebra $I / I \cap k K$ onto $I \mid K$, and, exactly as in $2.2,\|g \mid K\| \leqq$ $\|g+I \cap k K\|$, for $g$ in $I$. The reverse inequality follows by noting that if $f \in A$ peaks on $L \supset K$, where $L$ lies in a sufficiently small neighborhood of $K$, then $f^{n} g \in g+I \cap k K$, and $\|g+I \cap k K\| \leqq \lim \sup \left\|f^{n} g\right\| \leqq\|g \mid K\|+\varepsilon$.

A consequence of 2.5 is the fact that closed ideals of $A$ are relatively conjugate closed in the following sense. 
Corollary 2.6. Let $I$ be a closed ideal of $A, f \in I$. If the conjugate function $f$ is in $A$ then $\bar{f} \in I$.

For both $f \mid K$ and its conjugate $\bar{f} \mid K$ are in $A \mid K$, hence constant, so that both lie in $I \mid K$, for each $K$ in $\mathscr{K}$. So by $2.5\left(\mathrm{a}^{\prime}\right), \bar{f}$ is in $I$.

Let $\mathscr{M}_{A}$ be the set of multiplicative linear functions on $A$, and regard the elements of $\mathscr{M}_{A \mid K}$ as functionals on $A$ in the obvious fashion; then $\mathscr{M}_{A}$ is the (disjoint) union of the $\mathscr{M}_{A \mid K}, K \in \mathscr{K}$. This can be obtained from 2.5 , but we can argue more directly here as follows. For $\phi \in \mathscr{M}_{A}$, as is well known, there is a non-negative measure $\mu$ on $X$ representing $\phi: \phi(f)=\int f d \mu$ ( $\mu$ is necessarily of norm 1 since $\mu(1)=1)$. Let $\mu$ be in fact extreme in the set of all non-negative representing measures, and suppose

$$
\mu=a v+(1-a) \lambda, \quad 0<a<1, \quad v, \lambda \in \text { ball }\left(\phi^{-1}(0)\right)^{+} .
$$

Then $\mu(1)=1 \geqq\|v\|,\|\lambda\|$, implies $v(1)=\lambda(1)=1$ so that $\lambda, v \geqq 0$. Moreover $\lambda$ (and $v$ ) represent $\phi$ since $0=\lambda(f-\phi(f))=\lambda(f)-\phi(f) \lambda(1)=\lambda(f)-\phi(f), f \in A$. So $\lambda=v=\mu, \mu \in\left(\text { ball }\left(\phi^{-1}(0)\right)^{\perp}\right)^{e}$, and carrier $\mu$ is antisymmetric, contained in some $K$ in $\mathscr{K}$. Thus $\phi(k K)=0$, and $\phi \in \mathscr{M}_{A \mid K}$. The fact the $\mathscr{M}_{A \mid K}$ are pairwise disjoint follows from 2.3 : if $K_{1} \neq K_{2}$ are in $\mathscr{K}$ we have an $f$ in $A$ with $f\left(K_{1}\right)=1$, $\left|f\left(K_{2}\right)\right|<\varepsilon$. Thus $\phi$ in $\mathscr{M}_{A \mid K_{1}}$ implies $\phi(f)=1, \phi$ in $\mathscr{M}_{A \mid K_{2}}$ implies $|\phi(f)|<\varepsilon$.

$\mathscr{K}$ also has a bearing on the approximation of an element $g$ of $C(X)$ by elements of $A$. Indeed, let $\|g-A\|=\inf \{\|g-f\|: f \in A\},\|g|K-A| K\|=$ $\inf \{\|(g-f) \mid K\|: f \in A\}$. Then

$$
\|g-A\|=\max _{K \in \mathscr{K}}\|g|K-A| K\|
$$

(so that uniform approximation on $X$ is no worse than that attainable on maximal sets of antisymmetry). For since the normed space $A^{+}$is precisely the normed dual of $C(X) / A$,

$$
\|g-A\|=\sup \left\{|v(g)|: v \text { in ball } A^{\perp}\right\},
$$

and we have only to observe that $v \rightarrow v(g)$ assumes its maximum modulus over ball $A^{+}$at some extreme point $\mu$ of this set; $\mu$ is carried by some $K$ in $\mathscr{K}$ and thus, for $f$ in $A$,

$$
\|g-A\|=|\mu(g)|=|\mu(g-f)| \leqq\|\mu\| \cdot\|(g-f) \mid K\|
$$

so $\|g-A\| \leqq\|g|K-A| K\|$. Since the reverse inequality obtains trivially for any $K$, our assertion is clear. Moreover, for any closed ideal $I$ of $A$, since each element of (ball $\left.I^{+}\right)^{e}$ is carried by some $K$ in $\mathscr{K}$, our assertion holds with $I$ in place of $A$.

Finally we note one further property of the elements of $\mathscr{K}$ when $A$ is separating. Rudin [15] shows that if a compact set $K$ contains no perfect set then closed subalgebras of $C(K)$ are necessarily conjugate closed. Thus by $1.1(\mathrm{~b})$ every $K$ 
in $\mathscr{K}$ which contains more than one point contains a perfect set. (If $X=\mathscr{M}_{A}$ each $K$ is in fact connected, by a theorem of Silov [13, p. 168].)

3. Interpolation and $\mathscr{K}$. Let $F$ be a closed subset of $X$. The subalgebra $A$ of $C(X)$ is said to interpolate $C(F)$ if $A \mid F=C(F)$, and, given $A$, the general question of interpolation amounts to determining those $F$ for which this occurs. The present section is devoted to some results related to this question and to the bearing maximal sets of antisymmetry have on the problem; the results were suggested by an interpolation theorem of Bishop [5].

We shall still be concerned with orthogonal measures. Let $(A \mid F)^{\perp}$ denote the elements of $M(F)$ orthogonal to $A \mid F$ (or, equivalently, to its uniform closure $\left.(A \mid F)^{-}\right)$; these are of course precisely the elements of $A^{\perp}$ carried by $F$. For $v$ in $M(F)$ let $\left\|v-(A \mid F)^{\perp}\right\|=\inf \left\{\|v-\mu\|: \mu\right.$ in $\left.(A \mid F)^{\perp}\right\}$, the (quotient) norm in $M(F) /(A \mid F)^{+}$, and let $\left\|v-A^{+}\right\|=\inf \left\{\|v-\mu\|: \mu\right.$ in $\left.A^{+}\right\}$be that in $M(X) / A^{\perp}$ (we view $M(F)$ as a subset of $M(X)$ in the obvious fashion).

Our next result, which is fundamental to our considerations, was suggested by a proof by E. Nelson of Bishop's interpolation theorem [5]; it is a consequence of the fact $[9$, p. 488] that a continuous linear transformation from one Banach space to another has closed range if and only if its adjoint has. Both it and its corollary, 3.2, depend only on the hypothesis that $A$ is a closed subspace of $C(X)\left({ }^{2}\right)$.

THForem 3.1. Let $F$ be a closed subset of $X$. Then $A \mid F$ is closed in $C(F)$ if and only if, for some $c \geqq 1$,

$$
\left\|v-(A \mid F)^{\perp}\right\| \leqq c\left\|v-A^{\perp}\right\|, \quad v \text { in } M(F) .
$$

When (3.1) holds $A \mid F$ is topologically isomorphic with $A / k F$ and the constant $c$ dominates the norm of the isomorphism. Finally $c=1$ if and only if $A \mid F$ and $A / k F$ are isometric.

Proof. Consider the map $T: f \rightarrow f \mid F$ of $A$ into the uniform closure $(A \mid F)^{-}$ of $A \mid F$. We can identify the adjoint spaces of $A$ and $(A \mid F)^{-}$respectively with the quotient spaces $M(X) / A^{\perp}$ and $M(F) /(A \mid F)^{\perp}$, and clearly the adjoint $T^{*}$ takes the element $v-(A \mid F)^{\perp}$ of $M(F) /(A \mid F)^{\perp}$ into $v-A^{\perp} \in M(X) / A^{\perp}$. Obviously

$$
\left\|v-A^{\perp}\right\| \leqq\left\|v-(A \mid F)^{\perp}\right\|, \quad v \text { in } M(F),
$$

while $v$ in $A^{+}$implies $v \in(A \mid F)^{\perp}$ so $T^{*}$ is (1-1). Thus (3.1) is precisely equivalent to $T^{*}$ being topological. But with (3.2) available and $T^{*}(1-1)$ the last condition is equivalent to the closure of the range of $T^{*}$ by the open mapping theorem [9]. And by the result cited in our introductory remarks $T^{*}$ has closed range if and only if $T$ has, so the first assertion of 3.1 holds.

Now suppose (3.1) holds so that $T$ has closed range $A \mid F$, and is an open mapping by the open mapping theorem. Since $k F=\{f: f \in A, f(F)=0\}$ is just

(2) (3.1) and (3.2) have also been obtained by P. C. Curtis, Jr. and K. Hoffman. 
the nullity of $T$, the induced map $\tilde{T}$ of $A / k F$ onto $A \mid F$ is topological; on the other hand the adjoint $\tilde{T}^{*}$ coincides with $T^{*}$ (since we may identify $(A / k F) *$ with a subspace of $\left.A^{*}\right)$. Consequently bounds on the norms of $\tilde{T}$ and $\tilde{T}^{-1}$ can be read off from those of $\tilde{T}^{*}=T^{*}$ and $\left(\tilde{T}^{-1}\right)^{*}=\left(\tilde{T}^{*}\right)^{-1}=\left(T^{*}\right)^{-1}$; by (3.1) and (3.2) one has $\left\|\tilde{T}^{-1}\right\| \leqq c$, and $\|\tilde{T}\| \leqq 1$. Clearly $c=1$ if and only if $\tilde{T}^{-1}$ is an isometry, and all of the assertions of 3.1 are apparent.

The following corollary extends Bishop's interpolation theorem( $\left.{ }^{3}\right)$ [5].

Corollary 3.2. Let $F$ be a closed subset of $X$. Then $A \mid F=C(F)$ if and only if, for some $c \geqq 1$,

$$
\left\|\mu_{F}\right\| \leqq c\left\|\mu_{F^{\prime}}\right\|, \text { all } \mu \perp A .
$$

When (3.3) holds $c$ dominates the norm of the isomorphism of $A \mid F=C(F)$ onto $A / k F$.

Proof. $A \mid F=C(F)$ implies $(A \mid F)^{\perp}=0$ so that $\|v\| \leqq c\left\|v-A^{\perp}\right\|, v$ in $M(F)$. For $\mu$ in $A^{+}$and $v=\mu_{F}$ we therefore have $\left\|\mu_{F}\right\| \leqq c\left\|\mu_{F}-\mu\right\| \leqq c\left\|\mu_{F^{\prime}}\right\|$. so (3.3) holds.

On the other hand if (3.3) holds then $\mu \in(A \mid F)^{\perp}$ implies $\|\mu\|=\left\|\mu_{F}\right\| \leqq$ $c\left\|\mu_{F^{\prime}}\right\|=0$, and $(A \mid F)^{\perp}=0$, so $A \mid F$ is dense in $C(F)$. Since $(A \mid F)^{\perp}=0$, (3.1) becomes

$$
\|v\| \leqq c\left\|v-A^{+}\right\|, \quad v \text { in } M(F),
$$

so 3.2 will follow if (3.4) is established (since $A \mid F$ is then closed by 3.1 ). But for $v$ in $M(F)$ and $\mu$ in $A^{\perp}$

$$
\begin{aligned}
\|v-\mu\| & =\left\|(v-\mu)_{F}\right\|+\left\|(v-\mu)_{F^{\prime}}\right\|=\left\|v-\mu_{F}\right\|+\left\|\mu_{F^{\prime}}\right\| \\
& \geqq(1 / c)\left\|v-\mu_{F}\right\|+\left\|\mu_{F^{\prime}}\right\|
\end{aligned}
$$

where $c \geqq 1$ is the constant appearing in (3.3), so

$$
\begin{aligned}
\|v-\mu\| & \geqq(1 / c)\|v\|-(1 / c)\left\|\mu_{F}\right\|+\left\|\mu_{F^{\prime}}\right\| \\
& \geqq(1 / c)\|v\|-(1 / c) \cdot c\left\|\mu_{F^{\prime}}\right\|+\left\|\mu_{F^{\prime}}\right\|=(1 / c)\|v\| .
\end{aligned}
$$

Thus (3.4) holds, completing the proof.

We proceed next to some connections with maximal sets of antisymmetry.

THEOREM 3.3. Let $F$ be a closed Baire set (i.e., $a G_{\delta}$ ) in $X$ which is a union of maximal antisymmetric sets. Then $A \mid F$ is closed in $C(F)$, in fact isometric with $A / k F$.

Corollary 3.4. Let $f \in C(F)$ have $f|K \in A| K$ for all $K$ in $\mathscr{K}$ with $K \subset F$. Then $f \in A \mid F$.

(3) Which states that if $\mu_{F}=0$ for all $\mu+A$ then $C(F)=A \mid F$, with $A / k F$ and $C(F)$ isometric. 
For the collection of maximal antisymmetric sets for the closed (by 3.3) subalgebra $A \mid F$ of $C(F)$ is clearly $\{K: K \in \mathscr{K}, K \subset F\}$, and 1.1 applies. (Without 3.3 we could only conclude that $f$ was in $(A \mid F)^{-}$.) As an application of 3.4 note that if $F_{0}$ and $F_{1}$ satisfy the hypotheses of 3.3 and are disjoint then there is an $f$ in $A$ with $f\left(F_{0}\right)=0, f\left(F_{1}\right)=1$ (let $F=F_{0} \cup F_{1}$ ); in fact we have such an $f$ with $\|f\|<1+\varepsilon$ since $A \mid F$ and $A / k F$ are isometric by 3.3.

Proof of 3.3. Let $\mathscr{E}=\left(\text { ball } A^{+}\right)^{e}$ and $\mu \in$ ball $A^{\perp}$. By a recent result of Bishop and deLeeuw [2, Theorem 5.6] we may write

$$
\mu=\int_{\mathscr{E}} v \lambda(d v)
$$

where $\lambda$ is a non-negative measure of total variation 1 on a $\sigma$ ring of subsets of $\mathscr{E}$ and the integral exists in the $\left(\right.$ weak $\left.^{*}\right)$ sense that

$$
\mu(f)=\int_{\mathscr{E}} v(f) \lambda(d v)
$$

for each $f$ in $C(X)$. But (3.6), by usual monotoneity arguments, continues to hold for bounded Baire functions $f$ on $X$; consequently with $\phi_{F}$ the characteristic function of $F$,

$$
\mu\left(f \phi_{F}\right)=\int_{\mathscr{\delta}} v\left(f \phi_{F}\right) \lambda(d v), \quad f \in C(X)
$$

which we may write as

$$
\mu_{F}=\int_{\mathscr{E}} v_{F} \lambda(d v)
$$

Now for any $v$ in $\mathscr{E}, v_{F}=v$ or $v_{F}=0$, since carrier $v$ is contained in some $K$ in $\mathscr{K}$, and either $K \subset F$ or $K \cap F=\varnothing$ by hypothesis. Thus $v_{F}(f)=0$ for $f$ in $A$ and, by (3.8), $\mu_{F}(f)=0$. Consequently $\mu \perp A$ implies $\mu_{F} \perp A$, and so $\mu_{F} \in(A \mid F)^{\perp}$. For $v$ in $M(F)$ and $\mu \perp A$, we now have $\|v-\mu\| \geqq\left\|(v-\mu)_{F}\right\|=\left\|v-\mu_{F}\right\| \geqq$ $\left\|v-(A \mid F)^{\perp}\right\|$, so $\left\|v-A^{\perp}\right\| \geqq\left\|v-(A \mid F)^{\perp}\right\|$, and (3.1) holds with $c=1$. So 3.3 follows from 3.1 .

Our next result gives our connection between the interpolation problem and $\mathscr{K}$. We note first that the final assertion of 3.2 amounts to the fact that for any $g$ in $C(F)$ and $\varepsilon>0, g$ has an extension $f$ in $A$ with $\|f\|<(c+\varepsilon)\|g\|(\|g\|=$ $\sup |g(F)|$ of course).

THEOREM 3.5. Let $F$ be a closed Baire subset of $X, c$ a constant. Suppose that for each $K$ in $\mathscr{K}$ and every $g$ in $C(F \cap K)$ there is an $f$ in $A$ extending $g$ with $\|f\| \leqq c\|g\|$. Then for any $g$ in $C(F)$ and $\varepsilon>0$ there is an $f$ in $A$ extending $g$ with $\|f\| \leqq(c+\varepsilon)\|g\|$.

As we shall see later by an example (5.2) $c$ cannot be allowed to depend on $K$, i.e., in general for $A$ to interpolate $C(F)$ it is not sufficient to have $A$ interpolate 
$C(F \cap K)$ for each $K$ in $\mathscr{K}$. However it is sufficient for certain algebras (cf. 3.7 and 4.11 below).

Proof of 3.5. We again use the integral representation (3.5): for $\mu$ in $A^{\perp}$, $\mu /\|\mu\|=\int_{\mathscr{E}} v \lambda(d v)$, and $\mu_{F} /\|\mu\|=\int_{\mathscr{E}} v_{F} \lambda(d v)$, where $\lambda \leqq 0$ has total variation 1. By 3.2 our hypothesis implies that the constant $c$ has

$$
\left\|v_{F \cap K}\right\| \leqq c\left\|v_{(F \cap K)^{\prime}}\right\|, \text { all } v \perp A,
$$

for any $K$ in $\mathscr{K}$. Apply this in particular to $v$ in $\mathscr{E}$ and the $K$ in $\mathscr{K}$ which carries $v$ : since we have $v_{F}=v_{F \cap K}$ and $v_{(F \cap K)^{\prime}}=v_{F^{\prime} \cup K^{\prime}}=v_{F^{\prime}}$, we obtain $\left\|v_{F}\right\| \leqq c\left\|v_{F^{\prime}}\right\|$, for each $v$ in $\mathscr{E}$. Thus $(1+c)\left\|v_{F}\right\| \leqq c\left\|v_{F^{\prime}}\right\|+c\left\|v_{F}\right\|=c\|v\|=c$, since $\|v\|=1$ for $v$ in $\mathscr{E}$.

Now from our integral representation

$$
\frac{(1+c)\left\|\mu_{F}\right\|}{\|\mu\|} \leqq \bar{\int}(1+c)\left\|v_{F}\right\| \lambda(d v) \leqq \bar{\int} c \lambda(d v)=c
$$

(upper integrals being necessary since $v \rightarrow\left\|v_{F}\right\|$ is not appropriately measurable). So $\left\|\mu_{F}\right\| \leqq c\|\mu\|-c\left\|\mu_{F}\right\|=c\left\|\mu_{F^{\prime}}\right\|$ for all $\mu \perp A$, and 3.5 follows from 3.2 and the remark preceding 3.5.

As one class of algebras for which a strengthened form of 3.5 obtains one has the dirichlet algebras.

Definition 3.5.1. $A \subset C(X)$ is a dirichlet algebra on $X$ if $\operatorname{Re} A$ is dense in $C^{R}(X)[11 ; 17]$.

The next lemma could be obtained as a simple consequence of a later one (3.8); for later purposes we shall give its somewhat simpler direct proof.

Lemma 3.6. If $A$ is a dirichlet algebra on $X$ and $F$ is closed in $X$, then $A \mid F=C(F)$ if and only if $\mu \perp A$ implies $\mu_{F}=0$.

Clearly the second condition implies (3.3) with $c=1$, so we need only consider "only if." Suppose (3.3) holds, $\mu$ is orthogonal to $A$, say of norm 1 , and we choose a compact $K \subset F^{\prime}$ with $\left\|\mu_{F^{\prime} \mid K}\right\|<\varepsilon$. If we choose $g$ in $C^{R}(X)$ bounded by $\varepsilon$ and $1-\varepsilon$, and equal to $\varepsilon$ on $K$ and $1-\varepsilon$ on $F$ then we can approximate $\log g$ by $\operatorname{Re} f(f \in A)$ sufficiently well to insure that $\left\|e^{\operatorname{Re} f}-g\right\|<\varepsilon$; consequently $e^{\operatorname{Re} f}$ is bounded by 1 , exceeds $1-2 \varepsilon$ on $F$, and is less than $2 \varepsilon$ on $K$. Now for $h=e^{f} \in A,|h|=e^{\operatorname{Re} f}$ and $h \mu \perp A$, so by (3.3) we have

$$
\begin{aligned}
(1-2 \varepsilon)\left\|\mu_{F}\right\| \leqq\left\|(h \mu)_{F}\right\| & \leqq c\left\|(h \mu)_{F^{\prime}}\right\|=c\left\|h \mu_{F^{\prime} \backslash K}\right\|+c\left\|h \mu_{K}\right\| \\
& \leqq c \cdot 1 \cdot\left\|\mu_{F^{\prime} \backslash K}\right\|+c \cdot 2 \varepsilon\left\|\mu_{K}\right\| \\
& \leqq c \varepsilon+2 c \varepsilon=3 c \varepsilon,
\end{aligned}
$$

so $\mu_{F}=0$, which completes the proof.

Note that (since (3.3) holds with $c=1) A \mid F=C(F)$ for $A$ dirichlet implies $A \mid F$ and $A / k F$ are isometric, or that any $f$ in $A \mid F$ has an extension $g$ in $A$ with $\|g\| \leqq(1+\varepsilon)\|f\|$. Consequently 3.5 implies 
Corollary 3.7. Let $A$ be a dirichlet algebra on $X$, and $F$ a closed Baire subset of $X$. Then $A \mid F=C(F)$ if (and of course only if) $A \mid(F \cap K)=C(F \cap K)$ for all $K$ in $\mathscr{K}$.

For dirichlet algebras the condition (3.1) that $A \mid F$ be closed in $C(F)$ can be simplified, via the argument of 3.6.

Corollary 3.8. Let $A$ be dirichlet, $F$ a closed subset of $X$. Then $A \mid F$ is closed in $C(F)$ if and only if $\mu \perp A$ implies $\mu_{F} \perp A$. Consequently $A \mid F$ closed implies $A \mid F$ and $A / k F$ are isometric.

Since $\mu_{F} \perp A$ for $\mu \perp A$ implies $(A \mid F)^{\perp}=\left(A^{\perp}\right)_{F}\left(=\left\{\mu_{F}: \mu \in A^{\perp}\right\}\right)$ we have, for $v$ in $M(F),\left\|v-(A \mid F)^{\perp}\right\|=\left\|v-\left(A^{\perp}\right)_{F}\right\|=\left\|\left(v-A^{\perp}\right)_{F}\right\| \leqq\left\|v-A^{\perp}\right\|$, so that $A \mid F$ and $A / k F$ are isometric and $A \mid F$ closed.

Now if $A \mid F$ is closed then for some $c \geqq 1$

$$
\left\|v-(A \mid F)^{\perp}\right\| \leqq c\left\|v-A^{+}\right\|, \quad v \in M(F),
$$

so, for $\mu$ in $A^{+}$

$$
\left\|\mu_{F}-(A \mid F)^{\perp}\right\| \leqq c\left\|\mu_{F}-A^{\perp}\right\| \leqq c\left\|\mu_{F}-\mu\right\|=c\left\|\mu_{F^{\prime}}\right\| .
$$

Now fix $\mu$ in ball $A^{\perp}$ and choose $K \subset F^{\prime}$ and $f \in A$ as in the proof of 3.6; then $h=e^{f}$ is an invertible element of $A$ with $\|h\| \leqq 1,|h|>1-2 \varepsilon$ on $F$, and $|h|<2 \varepsilon$ on $K$. Applying (3.9) to $h \mu,\left\|h \mu_{F}-(A \mid F)^{\perp}\right\| \leqq c\left\|h \mu_{F^{\prime}}\right\| \leqq c\|h\|\left\|\mu_{F^{\prime} \backslash K}\right\|$ $+c \cdot 2 \varepsilon\left\|\mu_{K}\right\| \leqq c \varepsilon+2 c \varepsilon$. Since $h$ is invertible, and clearly $f(A \mid F)^{\perp} \subset(A \mid F)^{\perp}$ for $f$ in $A,(A \mid F)^{\perp}=h h^{-1}(A \mid F)^{\perp} \subset h(A \mid F)^{\perp} \subset(A \mid F)^{\perp}$. Thus $v$ in $(A \mid F)^{\perp}$ implies $v=h v^{\prime}, v^{\prime} \in(A \mid F)^{+}$, so $\left\|h \mu_{F}-v\right\|=\left\|h\left(\mu_{F}-v^{\prime}\right)\right\| \geqq(1-2 \varepsilon)\left\|\mu_{F}-v^{\prime}\right\|$ $\geqq(1-2 \varepsilon)\left\|\mu_{F}-(A \mid F)^{\perp}\right\|$, and $3 c \varepsilon \geqq(1-2 \varepsilon)\left\|\mu_{F}-(A \mid F)^{\perp}\right\|$. Consequently, $\mu_{F} \in(A \mid F)^{\perp}$, and the proof is complete.

3.8 has the following consequence.

Corollary 3.9. If $A$ is dirichlet on $X$ and $A \mid F_{i}$ is closed in $C\left(F_{i}\right), i=1,2$, then $A \mid\left(F_{1} \cup F_{2}\right)$ is closed in $C\left(F_{1} \cup F_{2}\right)$.

For if $\mu \perp A$ then $\mu_{F_{1} \cup F_{2}}=\mu_{F_{1}}+\mu_{F_{2}}-\mu_{F_{1} \cap F_{2}}$, where $\mu_{F_{i}} \perp A$, and thus $\mu_{F_{1} \cap F_{2}}=\left(\mu_{F_{1}}\right)_{F_{2}} \perp A$. (There is of course an analogous statement for a sequence $\left\{F_{n}\right\}$ of such sets having a closed union.)

Actually a somewhat more general assertion is true for an arbitrary $A$, directly from 3.1: if $\mu_{F_{1}} \perp A$ for all $\mu \perp A$, and $A \mid F_{2}$ is closed in $C\left(F_{2}\right)$, then $A \mid\left(F_{1} \cup F_{2}\right)$ is closed. For we have a $c \geqq 1$ for which $\left\|v-\left(A \mid F_{i}\right)^{+}\right\| \leqq c\left\|v-A^{+}\right\|, v$ in $M\left(F_{i}\right), i=1,2$, and if $v \in M\left(F_{1} \cup F_{2}\right)$ and $\mu$ is in $A^{+}$then $\|v-\mu\|=$ $\left\|v_{F_{1}}-\mu_{F_{1}}\right\|+\left\|v_{F_{2} \mid F_{1}}-\mu_{F_{1}^{\prime}}\right\| \geqq(1 / c)\left\|v_{F_{1}}-\left(A \mid F_{1}\right)^{+}\right\|+\left\|v_{F_{2} \mid F_{1}}-A^{+}\right\|$since $\mu_{F_{1}}$ and $\mu_{F_{1}^{\prime}}=\mu-\mu_{F_{1}}$ are orthogonal to $A$, so 


$$
\begin{aligned}
\|v-\mu\| & \geqq \frac{1}{c}\left\|v_{F_{1}}-\left(A \mid F_{1}\right)^{\perp}\right\|+\frac{1}{c}\left\|v_{F_{2} \backslash F_{1}}-\left(A \mid F_{2}\right)^{\perp}\right\| \\
& \geqq \frac{1}{c}\left(\left\|v_{F_{1}}-\left(A \mid\left(F_{1} \cup F_{2}\right)\right)^{\perp}\right\|+\left\|v_{F_{2} \backslash F_{1}}-\left(A \mid\left(F_{1} \cup F_{2}\right)\right)^{\perp}\right\|\right) \\
& \geqq \frac{1}{c}\left\|v-\left(A \mid\left(F_{1} \cup F_{2}\right)\right)^{\perp}\right\| .
\end{aligned}
$$

We might remark that 3.3, 3.4 and 3.5 hold with $A$ replaced by a closed ideal $I$ (take $I^{\perp}$ in place of $A^{\perp}$ in their proofs), since $\mu \in\left(\text { ball } I^{\perp}\right)^{e}$ implies carrier $\mu$ is an antisymmetric set of $A$ (cf. 2.5).

4. Interpolation with no increase in norm. Let $X$ be the unit circle $|z|=1$, and $\mathfrak{A}$ the subalgebra of $C(X)$ of functions having continuous extensions to $|z| \leqq 1$ analytic for $|z|<1$. If $F$ is a closed subset of $X$ of Lebesgue measure zero, Rudin [14] has shown that any $f$ in $C(F)$ has an extension $g$ in $\mathfrak{A}$ with $\|g\|=\|f\|$. Now by a well-known result of $\mathrm{F}$. and $\mathrm{M}$. Riesz any $\mu$ in $M(X)$ orthogonal to $\mathfrak{A}$ is absolutely continuous, so $\mu_{F}=0$; thus Bishop was able to apply his version of 3.2 to $\mathfrak{A}$ to obtain, more abstractly, a weaker result: for $\varepsilon>0, f$ has an extension $g_{\varepsilon}$ in $\mathfrak{U}$ with $\left\|g_{\varepsilon}\right\| \leqq(1+\varepsilon)\|f\|$. (Carleson [8] had obtained a slightly weaker result.)

In the present section we shall obtain a version of Rudin's result valid for dirichlet algebras (of which $\mathfrak{A}$ is the simplest non-trivial example). Indeed, it follows (cf. 4.5) from a construction due to Bishop [3] that if (i) $F$ is an intersection of peak sets, then for any $f$ in $A \mid F$ there is a $g$ in $A$ extending $f$ with $\|g\|=\|f\|$; and in Lemma 4.3 we shall see that for $A$ dirichlet (i) holds if $A \mid F$ is closed, in particular if $A \mid F=C(F)$. (For general $A$ the envisioned type of interpolation holds whenever $\mu_{F}=0$ for all $\mu \perp A$.)

Of course the norm preserving interpolation of $C(F)$ involved in Rudin's theorem can only be obtained when $A / k F$ and $C(F)$ are isometric; in 5.3 we give an example in which $A / k F$ and $C(F)$ are isometric, but norm preserving interpolation fails.

We begin with the following basic lemma, which was suggested by an argument of A. Browder and J. Wermer in [7].

Lemma 4.1. Let $F$ be a closed subset of $X, A$ a closed subalgebra of $C(X)$. If $A \mid F$ is closed in $C(F)$ then $(k F)^{\perp}=A^{\perp}+M(F)$. (Here $k F=\{f: f \in A, f(F)=0\}$, $(k F)^{\perp}$ is the set of orthogonal measures, and $M(F)$ is viewed as a subset of $M(X)$.)

Proof. Clearly $A^{\perp}+M(F) \subset(k F)^{\perp}$; on the other hand any $f$ in $C(X)$ orthogonal to $A^{\perp}+M(F)$ is in $A$ (being orthogonal to $A^{\perp}$ ) and vanishes on $F$ (being orthogonal to $M(F)$ ). Thus in the weak* topology of $M(X), A^{+}+M(F)$ is dense in $(k F)^{\perp}$, and so it suffices to prove $A^{\perp}+M(F)$ is weak* closed. But by a wellknown classical result $\left[9\right.$, p. 429] $A^{\perp}+M(F)$ is weak* closed if ball $\left(A^{\perp}+M(F)\right)$ is. 
So suppose $\left\{\mu_{\delta}+v_{\delta}\right\}$ is a net of elements of ball $\left(A^{\perp}+M(F)\right), \mu_{\delta} \in A^{\perp}, v_{\delta} \in M(F)$, and $\mu_{\delta}+v_{\delta} \rightarrow \mu$ weak* ${ }^{*}$, so $\|\mu\| \leqq 1$. By 3.1 there is a $c \geqq 1$ with

$$
\left\|v-(A \mid F)^{\perp}\right\| \leqq c\left\|v-A^{\perp}\right\| \text {, all } v \text { in } M(F) \text {, }
$$

and so there is a $\lambda_{\delta}$ in $(A \mid F)^{\perp}$ satisfying $\left\|v_{\delta}-\lambda_{\delta}\right\| \leqq 2 c\left\|v_{\delta}+\mu_{\delta}\right\| \leqq 2 c$. Consequently $\left\{v_{\delta}-\lambda_{\delta}\right\}$ has a weak* cluster point $v$ which lies in $M(F)$ since both $v_{\delta}$ and $\lambda_{\delta}$ are in $M(F)$, and $M(F)$ is weak* closed in $M(X)$.

But now $\left\{\mu_{\delta}+\lambda_{\delta}\right\}=\left\{\left(\mu_{\delta}+v_{\delta}\right)-\left(v_{\delta}-\lambda_{\delta}\right)\right\}$ has $\mu-v$ as a weak* cluster point; since $\mu_{\delta}$ and $\lambda_{\delta}$ are both in $A^{\perp}$, again weak* closed, $\mu-v \in A^{\perp}$, so $\mu \in A^{\perp}+M(F)$; since $\|\mu\| \leqq 1$, our proof is complete.

When $\mu_{F} \in A^{\perp}$ for all $\mu$ in $A^{+}$(as is the case for $A$ dirichlet (cf. 3.8)) we obtain $\left((k F)^{\perp}\right)_{F^{\prime}}=\left(A^{\perp}\right)_{F^{\prime}} \subset A^{\perp}$. This yields

CoRollary 4.2. Let $A$ be a dirichlet algebra on $X, F$ a closed subset of $X$ with $A \mid F$ closed in $C(F)$. Let $B=\{f: f \in A, f$ constant on $F\}, Y$ the factor space of $X$ obtained by identifying the points of $F$, and $\mathscr{B}$ the subalgebra of $C(Y)$ isomorphic (in the obvious fashion) to $B$. Then $\mathscr{B}$ is a dirichlet algebra on $Y$.

Thus, in particular, if $F$ is a closed set of measure zero on $|z|=1$, and $\mathscr{B}$ is the subalgebra of $\mathfrak{A}$ (above) of elements constant on $F, \mathscr{B}$ forms a dirichlet algebra on a circle with $F$ identified to a point.

Proof. Let $p$ be the point of $Y$ corresponding to the elements of $F$, and $\rho$ the natural map of $X$ onto $Y$. Then $\rho$ induces an isometry $\tilde{\rho}$ of $C(Y)$ into $C(X)$, taking $f$ into $f \circ \rho$, and thus (by Hahn-Banach) the adjoint $\tilde{\rho}^{*}$ takes $M(X)$ onto $M(Y)$. Clearly $\tilde{\rho} \mathscr{B}=B$, and, if $k\{p\}=\{f: f \in \mathscr{B}, f(p)=0\}$, then $\tilde{\rho} k\{p\}=k F$.

Now since $\tilde{\rho}^{*}$ is onto, $\tilde{\rho}^{*}$ maps $(k F)^{\perp}$ onto $(k\{p\})^{\perp}$. Indeed, $\tilde{\rho}^{*-1}(k\{p\})^{\perp}$ $\subset(k F)^{\perp}$, since $\tilde{\rho}^{*} \mu \perp k\{p\}$ and $f \in k F$ imply $f=\tilde{\rho} g, g \in k\{p\}$, and thus

$$
\mu(f)=\mu(\tilde{\rho} g)=\tilde{\rho}^{*} \mu(g)=0 .
$$

(For later use note that since $\tilde{\rho}^{*} M(F)$ is obviously $C \mu_{p}, C=$ complexes, $\mu_{p}=$ point mass at $p,(k\{p\})^{\perp}=\tilde{\rho}^{*}\left(\left(A^{\perp}\right)_{F}\right)+C \mu_{p}$.

Now in order to show $\mathscr{B}$ is dirichlet on $Y$ it suffices to show any $f$ in $C^{R}(Y)$ vanishing at $p$ can be uniformly approximated by $\operatorname{Re} g, g \in k\{p\}$. If this fails there is a real $\lambda$ in $M(Y)$ with $\lambda\{p\}=0, \lambda(f)=1$, and $\lambda(\operatorname{Re} g)=0$ (and so $\lambda(g)=0$ ) for all $g$ in $k\{p\}$. Evidently $\lambda$ is the image, under $\tilde{\rho}^{*}$, of a real measure $\mu$ on $X$ with $\mu=\mu_{F^{\prime}}$, i.e., $\mu_{F}=0$. Since $\tilde{\rho}^{*-1}(k\{p\})^{\perp}=(k F)^{\perp}, \mu \in(k F)^{\perp}$, and thus $\mu$ lies in $\left((k F)^{\perp}\right)_{F^{\prime}}=\left(A^{\perp}\right)_{F^{\prime}} \subset A^{\perp}$. So we have a real measure $\mu$ orthogonal to $A$, hence zero, whence $\lambda=\tilde{\rho}^{*} 0=0$, the desired contradiction.

Corollary 4.3. Let $A$ be a dirichlet algebra on $X, F$ a closed subset of $X$. Then $A \mid F$ is closed in $C(F)$ (if and) only if $F$ is an intersection of peak sets. (If $F$ is $a G_{\delta}$, "intersection of" is superfluous, as can be seen from the argument of 2.3.)

Proof. ("If" is of course contained in 2.2.) Suppose for the moment $X$ is 
metrizable, and $A \mid F$ closed. We form the space $Y$ and dirichlet algebra $\mathscr{B}$ of 4.2; since $C(Y)$ is separable, the compact space $Y$ is of course also metrizable. Let $p \in Y$ correspond to the identified elements of $F$. It follows from a result of Bishop [3, Theorem 2] that $p$ is a peak point of $\mathscr{B}$ if for every neighborhood $V$ of $p$ there is an $f$ in $\mathscr{B}$ with

$$
\|f\| \leqq 1,|f(p)|>\frac{3}{4},\left|f\left(V^{\prime}\right)\right|<\frac{1}{4} .
$$

Since $\mathscr{B}$ is dirichlet this is easily achieved; simply choose a positive $g$ in $C^{R}(X)$ of norm $7 / 8$ and value $7 / 8$ at $p$ which has $\left|g\left(V^{\prime}\right)\right|<1 / 8$, and approximate $\log g$ sufficiently well by $\operatorname{Re} h, h \in \mathscr{B}$, so that $\left|g-e^{\operatorname{Re} h}\right|<1 / 8$; then $f=e^{h} \in \mathscr{B}$ has the desired properties.

Thus there is an $f$ in $\mathscr{B}$ peaking at $p$; the corresponding element of $A$ of course peaks on $F$.

Now if $X$ is not metrizable we can still form $Y$ and $\mathscr{B}$ as before and for any neighborhood $V$ of $p$ obtain an $f$ in $\mathscr{B}$ satisfying (4.1). So given an initial neighborhood $V_{1}$ we choose a corresponding $f_{1}$ in $\mathscr{B}$ and let $V_{2}=\left\{y: y \in Y,\left|f_{1}(y)-f_{1}(p)\right|\right.$ $<1 / 2\}$, choosing $f_{2}$ in $\mathscr{B}$ satisfying (4.1) for $V=V_{2}$; having chosen $V_{2}, \cdots, V_{n-1}$, $f_{1}, \cdots, f_{n-1}$ we let

$$
V_{n}=\left\{y: y \in Y,\left|f_{i}(y)-f_{i}(p)\right|<\frac{1}{n}, i \leqq n-1\right\}
$$

and choose $f_{n}$ in $\mathscr{B}$ satisfying (4.1) for $V=V_{n}$. (Note that $V_{n} \subset V_{1}$ for $n$ sufficiently large.)

Let $Z$ be the set of equivalence classes of $Y$ corresponding to $y_{1} \sim y_{2} \Leftrightarrow f_{n}\left(y_{1}\right)$ $=f_{n}\left(y_{2}\right)$, all $n$; let $\tilde{f}_{n}(z)=f_{n}(y)$ for $y \in z$, and endow $Z$ with the least fine topology rendering all the $\tilde{f}_{n}$ continuous. Then $Z$ is a compact metrizable space for which the closed subalgebra $\mathscr{B}_{1}$ of $\mathscr{B}$ generated by $\left\{f_{1}, f_{2}, \cdots\right\}$ is isomorphic to the subalgebra $\tilde{\mathscr{B}}_{1}$ of $C(Z)$ generated by $\left\{\tilde{f}_{1}, \tilde{f}_{2}, \cdots\right\}$ under a correspondence $f \leftrightarrow \tilde{f}$ for which $\tilde{f}(\tilde{y})=f(y)$, where $\tilde{y}$ is the equivalence class of $y$. Moreover the neighborhoods

$$
\tilde{V}_{n}=\left\{z: z \in Z,\left|\tilde{f}_{i}(z)-\tilde{f}_{i}(\tilde{p})\right|<\frac{1}{n}, i \leqq n-1\right\}
$$

clearly form a base of neighborhoods of $\tilde{p}$ so that for each neighborhood $\tilde{V}$ of $\tilde{p}$ there is an $\tilde{f}$ (some $\tilde{f}_{n}$ ) satisfying (4.1). Thus Bishop's theorem [3, Th. 2] applies to yield an $\tilde{f}$ in $\tilde{\mathscr{B}}$ peaking at $\tilde{p}$; the corresponding $f$ in $\mathscr{B}_{1}$ clearly peaks on a set lying between $\bigcap_{n=2}^{\infty} V_{n}$ and $F$, thus between $V_{1}$ and $F$, since $V_{n} \subset V_{1}$ for $n$ sufficiently large. Since $V_{1}$ is an arbitrary neighborhood of $p$, the proof is complete.

4.3 and 3.9 combine to yield the rather interesting

Corollary 4.4. Let $A$ be a dirichlet(4) algebra on $X$, and let $F_{1}$ and $F_{2}$ be intersections of peak sets. Then $F_{1} \cup F_{2}$ is an intersection of peak sets. (If $X$ is metric, "intersection of" can be omitted, as in 4.3.)

(4) This restriction is superfluous; cf. the final remarks of $\$ 5$. 
Our generalized Rudin theorem will follow from 4.3 and the following lemma (based upon an argument of Bishop [3, p. 631]).

LEMMA 4.5. Let $A$ be a closed subalgebra of $C(X)$ and $F$ an intersection of peak sets. Then any $f$ in $A \mid F$ has an extension $g$ in $A$ with $\|g\|=\|f\|(=\sup |f(F)|)$. (When $F$ is a peak set we can in fact assert that $|g(x)|<\|f\|$ for $x \notin F$.)

Proof. Let $\|f\|=1$. Since $A / k F$ and $A \mid F$ are isometric by 2.2 , choose an $h$ in $A$ extending $f$ with $\|h\|<3 / 2$. Then $\{x:|h(x)| \leqq 1\}$ is $a G_{\delta}$ containing $F$, say $\bigcap_{n=1}^{\infty} V_{n}, V_{n}$ open, $F \subset V_{n}$. But each $V_{n}$ contains a peak set $F_{n}$ containing $F$, so $F_{0}=\bigcap F_{n}$ is a peak set containing $F$ on which $|h| \leqq 1$. Let $k \in A$ peak on $F_{0}$, and set

$$
K_{n}=\left\{x: 1+2^{-n-1} \leqq|h(x)| \leqq 1+2^{-}\right\}, \quad n=1,2, \ldots .
$$

Since $K_{n} \cap F_{0}=\varnothing$ we can choose an integer $p_{n}$ for which $\left|k^{p_{n}}\right|<1 / 2^{2 n}$ on $K_{n}$. Now set

$$
g=\sum_{n=1}^{\infty} 2^{-n} k^{p_{n}} h
$$

which is the desired element of $A$. For $g|F=h| F=f$, while certainly $|g(x)|$ $\leqq 1$ if $|h(x)| \leqq 1$ since $\|k\|=1$. On the other hand if $|h(x)|>1$ then (since $\|h\|<3 / 2) x \in K_{m}$ for some $m \geqq 1$ and so

$$
\begin{aligned}
|g(x)| & \leqq|h(x)| \cdot\left(\sum_{n \neq m} 2^{-n}+2^{-m} \cdot 2^{-2 m}\right) \\
& \leqq\left(1+2^{-m}\right)\left(1-2^{-m}+2^{-3 m}\right) \\
& =\left(1-2^{-2 m}\right)+\left(1+2^{-m}\right) 2^{-3 m} \\
& =1-2^{-2 m}+2^{-3 m}+2^{-4 m}=1-\frac{\left(2^{2 m}-2^{m}-1\right)}{2^{4 m}}<1,
\end{aligned}
$$

and thus $\|g\| \leqq 1=\|f\|$.

For the final assertion note that when $F$ is a peak set we can take $F_{0}=F$, and thus replace $g$ by $g k$, where $k$ (as above) peaks on $F_{0}$.

By 4.3 and 4.5 we have

THEOREM 4.6. Let $A$ be a dirichlet algebra on $X$, and let $F$ be a closed subset of $X$ with $A \mid F$ closed in $C(F)$. Then any $f$ in $A \mid F$ has an extension $g$ in $A$ with precisely the same norm as $f$.

Finally if $F$ is a $G_{\delta}$ (in particular, if $X$ is metric) then $g$ can be chosen so that $|g(x)|<\|f\|, x \notin F$.

Of course the hypothesis of 4.6 can be restated in terms of measures by 3.8. For comparison with Rudin's theorem (recalling the F. and M. Riesz theorem) let us state a special case.

Corollary 4.7. Let $A$ be dirichlet on $X, F$ a closed subset of $X$. If $\mu_{F}=0$ for all $\mu \perp A$ (i.e., if $A \mid F=C(F)$ ) then any $f$ in $C(F)$ has an extension $g$ in $A$ of the same norm as $f$. 
Actually most of our results on dirichlet $A$, in particular 4.3, 4.6 and 4.7, apply to a slightly wider class of algebras $A$ : those for which $A \mid F$ closed in $C(F)$ implies $\mu_{F} \perp A$ for $\mu \perp A$. This will become apparent in the proof of

THEOREM 4.8. Let $A$ be a closed separating subalgebra of $C(X)$ containing the constants and $F$ a closed subset of $X$.

(a) If $\mu_{F} \perp A$ for all $\mu \perp A$ then $A \mid F$ is closed and any $f$ in $A \mid F$ has an extension $g$ in $A$ of the same norm as $f$.

(b) If $\mu_{F}=0$ for all $\mu \perp A$ then any $f$ in $C(F)$ has an extension $g$ in $A$ of the same norm as $f$.

In each case $F$ is an intersection of peak sets of $A$ (and a peak set if it is a $G_{\delta}$ ).

Of course (b) follows from (a) since $A \mid F$ must then be dense in $C(F)$. To obtain (a), note first that $A / k F$ and $A \mid F$ are isometric (and so $A \mid F$ closed) as in 3.8. Consequently 4.1 implies $(k F)^{\perp}=A^{\perp}+M(F)=\left(A^{\perp}\right)_{F^{\prime}}+M(F)$. We shall (essentially) complete the proof by proving the final assertion.

Let $B=\{f: f \in A, f$ constant on $F\}$, let $Y$ be obtained from $X$ by identifying the points of $F$ to a single point $p$, and let $\mathscr{B}$ be the subalgebra of $C(Y)$ obtained from $B$ as before. Let $k\{p\}=\{f: f \in \mathscr{B}, f(p)=0\}$, and let $\rho$ be the canonical map of $X$ onto $Y$, with $\tilde{\rho}$ the induced $\operatorname{map} f \rightarrow f \circ \rho$ of $C(Y)$ into $C(X), \tilde{\rho}^{*}: M(X) \rightarrow M(Y)$ its adjoint.

Now exactly as in 4.2 we have

$$
(k\{p\})^{+}=\tilde{\rho}^{*}\left(\left(A^{\perp}\right)_{F^{\prime}}\right)+C \mu_{p}
$$

where $C=$ complexes, $\mu_{p}=$ point mass at $p$. Since $\mathscr{B}=k\{p\}+C, \mathscr{B}^{\perp}$ consists of just the elements of $(k\{p\})^{\perp}$ which are also orthogonal to the constant functions on $Y$. But $\tilde{\rho}^{*}\left(\left(A^{\perp}\right)_{F^{\prime}}\right)(1)=\left(A^{\perp}\right)_{F^{\prime}}(1)=0$ since $\left(A^{\perp}\right)_{F^{\prime}} \subset A^{\perp}$, while $c \mu_{p}(1)=0$ only if $c=0$, so $\mathscr{B}^{+}=\tilde{\rho}^{*}\left(\left(A^{+}\right)_{F^{\prime}}\right)$. In particular then, for $\mu$ in $\mathscr{B}^{+}, \mu\{p\}=0$.

We now note that $\mathscr{B}$ is separating on $Y$ since we can show $k F$ separates distinct elements of $X \backslash F$, and for $x \in X \backslash F$ there is an $f$ in $k F$ with $f(x) \neq 0$. Indeed if the last assertion fails, $\mu_{x}$, the point mass at $x$, lies in $(k F)^{\perp}=\left(A^{\perp}\right)_{F^{\prime}}+M(F)$, so $\mu_{x}=\left(\mu_{x}\right)_{F^{\prime}} \in\left(A^{\perp}\right)_{F^{\prime}} \subset A^{\perp}$, which is impossible. Similarly failure of the other assertion leads to $\mu_{x}-\mu_{x^{\prime}} \in A^{\perp}, x, x^{\prime}$ distinct elements of $X \backslash F$.

Now suppose for the moment that $X$ is metrizable, so that $Y$ also is. Then it suffices to show $p$ is a peak point of $\mathscr{B}$; for then $F$ is correspondingly a peak set of $A$, and 4.5 applies to yield (a). But if $p$ is not a peak point of the separating algebra $\mathscr{B}$, by $[3$, Th. 3] we have a measure $\mu$ with $\mu\{p\}=0$ which represents $p: f(p)=$ $\int f d \mu, f \in \mathscr{B}$. This implies $\mu_{p}-\mu$ is a measure orthogonal to $\mathscr{B}$ which does not vanish on $\{p\}$, and this, as we saw, is impossible.

This general case is covered by the deeper results of [2]. By 4.5 it suffices to show $F$ is an intersection of peak sets of $A$, and thus to show $p$ is an intersection of peak sets of $\mathscr{B}$. In the terminology of [2], the last amounts to saying $p$ satisfies condition II, and if this fails to hold, by $[2,6.5]$ there is a positive Baire measure $\mu$ on $Y$, $\mu \neq \mu_{p}$, for which $\mu-\mu_{p}$ is orthogonal to $\mathscr{B}$. Replacing these Baire measures 
by their regular Borel extensions we have of course the same situation: $\mu-\mu_{p}$ is a nonzero element of $M(Y)$ orthogonal to $\mathscr{B}$. But since $\mu \geqq 0, \mu\{p\} \neq 1$, and thus $\left(\mu-\mu_{p}\right)(\{p\}) \neq 0$ despite our earlier conclusion; this contradiction thus completes the proof.

We can now point out a class of algebras, which includes dirichlet algebras, for which interpolation of $C(F)$ implies the norm preserving interpolation of Rudin's theorem.

Definition 4.8.1. $A \subset C(X)$ is said to approximate in modulus on $X$ if, for every positive continous $g$ on $X$ and $\varepsilon>0$, there is an $f$ in $A$ with $|g-| f||<\varepsilon$.

To justify our remark, note that by precisely the proof of 3.6 (taking the $f$ of 4.8.1 as the $h$ used in 3.6) one proves

Lemma 4.9. If $A$ approximates in modulus on $X$ and $F$ is closed in $X$, then $A \mid F=C(F)$ if and only if $\mu \perp A$ implies $\mu_{F}=0$.

Consequently by 4.8 (b) we have

THEOREM 4.10. If $A$ approximates in modulus on $X$, and $F$ is closed in $X$, then the following are equivalent:

(a) $\mu \perp A$ implies $\mu_{F}=0$,

(b) $A \mid F=C(F)$,

(c) any $f$ in $C(F)$ has an extension $g$ in $A$ of the same norm as $f$.

Exactly as in 3.7 we obtain

Corollary 4.11. If $A$ approximates in modulus on $X$, and $F$ is a closed Baire subset of $X$, then $A \mid F=C(F)$ if and only if $A \mid(F \cap K)=C(F \cap K)$ for all $K$ in $\mathscr{K}$.

Approximation in modulus is not strong enough to allow us to obtain the analogue of 3.8 (the $h$ used there has to be invertible); consequently we cannot apply 4.8(a). In order to point up how wide this class of algebras is, we note

LEMMA 4.12. Let $\mathscr{U}$ be the set of unimodular elements of $A$, i.e. $\{f: f \in A,|f|=1$ on $X\}$. If $\mathscr{U}$ separates $X$ then $A$ approximates in modulus on $X$.

Indeed we have only to note that $\overline{\mathscr{U}} A=\{\bar{u} f: u \in \mathscr{U}, f \in A\}$ (where the bar denotes conjugation) is an algebra: it is clearly closed under multiplication, while $\bar{u}_{1} f_{1}$ $+\bar{u}_{2} f_{2}=\bar{u}_{1} \bar{u}_{2}\left(u_{2} f_{1}+u_{1} f_{2}\right)$ shows it is closed under addition. Since it contains the algebra generated by $\mathscr{U}$ and $\overline{\mathscr{U}}$, which is conjugate closed, and separating by hypothesis, $\overline{\mathscr{U}} A$ is dense in $C(X)$ by Stone-Weierstrass. So for $\varepsilon>0$ and $g$ any positive element of $C^{R}(X)$ we have $|g-\bar{u} f|<\varepsilon$ for appropriate $u, f$ in $A$, whence $|g-| \bar{u} f||=|g-| f||<\varepsilon$.

As a particular example (where 4.12 is more transparent directly) take $A$ to be the subalgebra of the continuous functions on the torus $T^{2}$ which are boundary values of functions continuous on the closed bicylinder $\{(z, w):|z| \leqq 1,|w| \leqq 1\}$ and analytic on the interior. (In this connection we can see that the difficulty in 
obtaining specific applications of our version of Rudin's theorem (4.10 or 4.7) will reside in finding the appropriate version of the F. and M. Riesz theorem, i.e., a result identifying the $F$ for which 4.10 (a) holds.)

Finally we turn to a result which is concerned with interpolation, but not of the norm preserving variety; we include it here since part of its proof was suggested by Rudin's proof in [14].

If $p$ is a peak point of $A$, any $\mu$ orthogonal to $A$ vanishes on $\{p\}$, since if $f$ peaks at $p$ then $f^{n} \mu \perp A$, and so, by dominated convergence, $\phi_{\{p\}} \mu \perp A$, and $\mu\{p\}=0$. Thus for $F$ closed, countable, and consisting of peak points (so that $\mu_{F}=0$ for $\mu \perp A$ ), it was known from Bishop's interpolation theorem that $A$ interpolates $C(F)$. Our final result makes a stronger assertion: we need only know that each pair of distinct elements of $F$ are not equivalent in the sense of Gleason [11] ( $x, y$ in $X$ are by definition equivalent in Gleason's sense if $\sup \{|f(x)-f(y)|: f \in A,\|f\|<1\}$ $<2$, i.e. if $\mu_{x}-\mu_{y}$ provides a functional of norm $<2$ on $A$ ). What we need to suppose about $F$ in addition, rather than countability, is that the elements of $M(F)$ are discrete, and this holds if $\left({ }^{5}\right)$ we assume $F$ is scattered, i.e., contains no nonempty perfect subsets (which amounts to countability in case $X$ is metric). For we can always split $\mu$ in $M(F)$ into a discrete and continuous part, while a continuous measure clearly has for its carrier a set without isolated points, i.e., a perfect set.

THEOREM 4.13. Let $F$ be a closed scattered subset of $X$. If $x, y \in F, x \neq y$ imply $x$ and $y$ are not equivalent in Gleason's sense (i.e., if

$$
\sup \{|f(x)-f(y)|: f \in A,\|f\| \leqq 1\}=2)
$$

then $A / k F$ and $A \mid F=C(F)$ are isometrically isomorphic.

The (second) hypothesis can be stated another way in terms of measures: $\left\|\mu_{x}-\mu_{y}-A^{+}\right\|=2\left(=\left\|\mu_{x}-\mu_{y}\right\|\right)$. This is of course implied by the conclusion, which in these terms assumes the form

$$
\left\|\sum_{n=1}^{\infty} \lambda_{n} \mu_{x_{n}}-A^{\perp}\right\|=\left\|\sum_{n=1}^{\infty} \lambda_{n} \mu_{x_{n}}\right\|
$$

for all $\left\{\lambda_{1}, \lambda_{2} \ldots\right\}$ in $e_{1}$ and distinct $x_{n}$ in $F$.

To proceed to the proof, note that for distinct $x$ and $y$ in $F$ we have an $f$ in $A$ of norm $<1$ with $f(x), f(y)$ arbitrarily close to $1,-1$ respectively. Since( $\left(^{6}\right)$ $z \rightarrow(z-f(y)) /(1-\overline{f(y)} z)$ is analytic on $|z| \leqq 1$, hence on a neighborhood of the spectrum of $f$,

$$
g=\frac{f-f(y)}{1-\overline{f(y) f}}
$$

(5) And only if as well, since any perfect compact space carries nonzero continuous measures, cf. [12].

(6) At several points in the following argument we shall make use of the fact that the spectrum of $f \in A$ is contained in its boundary (which lies in $f(X)$ ) plus the bounded components of the complement of the boundary, and also that for $\phi$ analytic on a neighborhood of the spectrum, $\phi \circ f \in A$, cf. [13]. 
is an element of $A$ of norm $<1$ with $g(y)=0$ and

$$
g(x)=\frac{f(x)-f(y)}{1-\overline{f(y)} f(x)}
$$

as close as we please to $(f(x)-(-1)) /(1-(-1) f(x))=1$.

Now given $n$ distinct points $x_{1}, \cdots, x_{n}$ in $F$ we can choose $g_{2}, \cdots, g_{n}$ in $A$ with $\left\|g_{i}\right\|<1, g_{i}\left(x_{i}\right)=0$, and $g_{i}\left(x_{1}\right)$ arbitrarily close to 1 . Thus $g=\prod_{2}^{n} g_{i}$ is an element of $A$ with $\|g\|<1, g\left(x_{2}\right)=\cdots=g\left(x_{n}\right)=0$, and we are free to choose $g$ so that $g\left(x_{1}\right)$ is as close as we please to 1 . Let $h=\left(g-g\left(x_{1}\right)\right) /\left(\overline{g\left(x_{1}\right)} g-1\right)$; again $h \in A,\|h\|<1$, since $z \rightarrow\left(z-g\left(x_{1}\right)\right) /\left(\left(\overline{g\left(x_{1}\right)} z-1\right)\right.$ is a conformal self map of the unit disc. And $h\left(x_{1}\right)=0$ while we can make $h\left(x_{2}\right)=\cdots=h\left(x_{n}\right)=g\left(x_{1}\right)$ arbitrarily close to 1 .

Consequently we have elements $h_{1}, \cdots, h_{n}$ of $A$ with $\left\|h_{i}\right\|<1, h_{i}\left(x_{i}\right)=0$, and if $i \neq j, h_{i}\left(x_{j}\right)$ arbitrarily close to 1 . Let

$$
\phi(z)=\frac{1-z}{1+z}
$$

which takes $|z|<1$ onto $\operatorname{Re} z>0$. Since -1 is not in the spectrum of $h_{i}, \phi$ is analytic on a neighborhood of this set, and $\phi \circ h_{i} e \in A, \operatorname{Re} \phi \circ h_{i}>0$. Note that $\phi \circ h_{i}\left(x_{i}\right)=1$, while by an appropriate choice of $h_{1}, \cdots, h_{n}$ we can make $\phi \circ h_{i}\left(x_{j}\right)$ as close as we please to $\phi(1)=0, i \neq j$.

Now suppose $f \in C\left(\left\{x_{1}, \cdots, x_{n}\right\}\right)$ is given, $\|f\|<1$. For each $j$ choose a conformal self map $\psi_{j}$ of the Riemann sphere which takes $\operatorname{Re} z \geqq 0$ onto itself, 0 into 0 , and 1 into $\phi\left(f\left(x_{j}\right)\right)\left(\left|f\left(x_{j}\right)\right|<1\right.$ so $\operatorname{Re} \phi\left(f\left(x_{1}\right)\right)>0$ and $\psi_{j}$ exists). Since $\psi_{j}$ has its singularity on $\operatorname{Re} z=0$ (or at $\infty$ ) and $\operatorname{Re} \phi \circ h_{j}>0, \psi_{j} \circ \phi \circ h_{j} \in A$ and $\operatorname{Re} \psi_{j} \circ \phi \circ h_{j}>0$. Thus if we form

$$
h=\phi^{-1}\left(\sum_{j} \psi_{j} \circ \phi \circ h_{j}\right),
$$

$h$ is an element of $A$ of norm $<1$ since $\operatorname{Re} \sum \psi_{j} \circ \phi \circ h_{j}>0$ and $\phi^{-1}$ is analytic on $\operatorname{Re} z \geqq 0$. Moreover we can make $\phi \circ h_{j}\left(x_{i}\right)$ close to 0 for $i \neq j$, so $\Sigma_{j} \psi_{j} \circ \phi \circ h_{j}\left(x_{i}\right)$ can be made arbitrarily close to $\psi_{i} \circ \phi \circ h_{i}\left(x_{i}\right)=\psi_{i}(1)$ $=\phi \circ f\left(x_{i}\right)$, and $h\left(x_{i}\right)$ will approximate $f\left(x_{i}\right)$ arbitrarily well, $i=1, \ldots, n$.

So for $\varepsilon>0$ we have a $g_{1}$ in $A$ with $\left\|g_{1}\right\|<1$, sup $\left|\left(f-g_{1}\right)\left(x_{i}\right)\right|<\varepsilon$. Similarly we have $g_{2}$ in $A$ with $\left\|g_{2}\right\|<\varepsilon$, sup $\left|\left(f-g_{1}-g_{2}\right)\left(x_{i}\right)\right|<\varepsilon^{2}$, and, iterating, we have $g_{n}$ in $A$ with $\left\|g_{n}\right\|<\varepsilon^{n-1}$, sup $\left|\left(f-\sum_{1}^{n} g_{i}\right)\left(x_{i}\right)\right|<\varepsilon^{n}$. Thus $g=\sum_{1}^{\infty} g_{i}$ is an element of $A$ of norm $<1 /(1-\varepsilon)$ which extends our element $f$ of $C\left(\left\{x_{1}, \cdots, x_{n}\right\}\right)$. Since $f$ was any element of norm $<1$ we have shown $A / k\left\{x_{1}, \cdots, x_{n}\right\}$ and $C\left(\left\{x_{1}, \cdots, x_{n}\right\}\right)$ to be isometric. By 3.2 this implies

$$
\left\|\mu_{\left\{x_{1}, \ldots, x_{n}\right\}}\right\| \leqq\left\|\mu_{\left\{x_{1}, \ldots, x_{n}\right\}^{\prime}}\right\| \text {, all } \mu \perp A .
$$
Then

Now given $\varepsilon>0$ and $\mu \perp A$ choose $x_{1}, \cdots, x_{n}$ in $F$ for which $\left\|\mu_{F \backslash\left\{x_{1}, \ldots, x_{n}\right\}}\right\|<\varepsilon$.

$$
\left\|\mu_{F}\right\| \leqq\left\|\mu_{\left\{x_{1}, \ldots, x_{n}\right\}}\right\|+\varepsilon \leqq\left\|\mu_{\left\{x_{1}, \ldots, x_{n}\right\}^{\prime}}\right\|+\varepsilon \leqq\left\|\mu_{F^{\prime}}\right\|+2 \varepsilon
$$


since $\varepsilon>0$ is arbitrary, $\left\|\mu_{F}\right\| \leqq\left\|\mu_{F^{\prime}}\right\|$, and since $\mu \perp A$ is arbitrary, our theorem follows from 3.2.

5. Some examples. Let $C$ denote the complex numbers, $D$ the unit disc $\{z:|z| \leqq 1\}$ and $T$ the unit circle $\{z:|z|=1\}$ in $C$. Let $I$ denote the interval $[0,1]$ of the real line.

5.1. We begin with a simple example in which the decomposition space $\mathscr{K}$ yields is not Hausdorff. In $I \times D$ let $X$ be the subset consisting of all $(r, z)$ with $|z| \geqq 1-r / 2$ (a cylinder with a conical section removed). Let $A$ be the (closed) subalgebra of $C(X)$ formed by those $f$ with $z \rightarrow f(r, z)$ analytic for $1-r / 2<|z|$ $<1$, for each $r>0$, and let $X_{r}=\{(r, z):(r, z) \in X\}$, so $X_{0}$ is a circle, $X_{r}$, for $r>0$, a plane annulus.

Now clearly $X_{r}$, for $r>0$, is a set of antisymmetry for $A$, which, since $f_{1}:(r, z) \rightarrow r$ is in $A$, is obviously maximal. On the the other hand each point of $X_{0}$ forms a maximal set of antisymmetry. Indeed both $(r, z) \rightarrow z$ and $(r, z) \rightarrow z^{-1}$ are in $A$ so that $A \mid X_{0}$ contains a dense (by Stone-Weierstrass) self adjoint subalgebra of $C\left(X_{0}\right)$.

Consequently the decomposition space $Y$ we obtain from $\mathscr{K}$ in this example can be identified with a space formed from $X_{0} \cup(0,1]$ in which every neighborhood of $x_{0} \in X_{0}$ includes some subinterval $(0, \varepsilon)$ of $(0,1]$, so $Y$ is not Hausdorff.

It might be noted that the decomposition space $Y$ obtained from $\mathscr{K}$ is Hausdorff if and only if $\mathscr{K}$ coincides with the (a priori less fine) collection of sets of constancy of the real valued elements of $A$ (i.e., the equivalence classes corresponding to $x_{1} \sim x_{2} \Leftrightarrow f\left(x_{1}\right)=f\left(x_{2}\right)$, all real $f$ in $A$ ), and thus if and only if Šilov's decomposition (cf. $[13,15]$ ) coincides with Bishop's. Indeed "if" follows from the fact that Silov's decomposition is Hausdorff[13]. On the other hand if $Y$ is Hausdorff (so $C^{R}(Y)$ separates $Y$ ) and $\rho$ is the canonical map of $X$ onto $Y$, then $\tilde{\rho}: f \rightarrow f \circ \rho$ maps $C^{R}(Y)$ into $A$ (since $f \circ \rho$ is constant on each $K$ in $\mathscr{K}$ ); since any real $f$ in $A$ is constant on each $K$ in $\mathscr{K}$, and thus is $g \circ \rho$ for some $g$ in $C^{R}(Y)$, $A \cap C^{R}(X)=\tilde{\rho} C^{R}(Y)$. Consequently the sets of constancy of $A \cap C^{R}(X)$ are precisely the $\rho^{-1}(y), y \in Y$ (i.e., the elements of $\mathscr{K}$ ).

5.2. Our next example shows interpolation of $C(F \cap K)$ for every $K$ in $\mathscr{K}$ is not sufficient to yield interpolation of $C(F)$ (cf. 3.5). Let $X$ be the subset of $I \times D$ formed by the circles $\{0\} \times T,\{1 / n\} \times T, n=1,2, \cdots$ (or, more concisely, $E \times T$, where $E=\{0,1,1 / 2,1 / 3, \cdots\})$ along with $F=(E \times\{0\}) \cup\{(r, r): r \in E\}$. (Thus $X$ lies in the planes $r=1 / n$ (or $=0)$ and meets each such plane in a circle plus (at most) two points interior to the circle.) Let $A$ be the subalgebra of $C(X)$ of those $f$ for which $z \rightarrow f(1 / n, z)$ and $z \rightarrow f(0, z)$ have continuous extensions to $|z| \leqq 1$ analytic for $|z|<1$. As is easily seen $\mathscr{K}$ consists precisely of the plane sections, viz: $(\{0\} \times T) \cup\{(0,0)\}$ and $(\{1 / n\} \times T) \cup\{(1 / n, 0),(1 / n, 1 / n)\}$ for $n=1,2, \cdots$.

Now $F$ is closed in $X$ and, since $F \cap K$ contains at most two points for each $K$ in $\mathscr{K}, A \mid(F \cap K)=C(F \cap K)$. On the other hand $A \mid F \neq C(F)$. Indeed let 
$f \in C(F)$ be defined by $f(r, 0)=0, f(r, r)=\sqrt{r}, r \in E$. Suppose $g \mid F=f, g$ in $A$. Then since $g(1 / n, 0)=f(1 / n, 0)=0$, by Schwarz's lemma $g(1 / n, 1 / n)=\sqrt{1 / n}$ $\leqq\|g\| \cdot 1 / n$, and $\sqrt{n} \leqq\|g\|$ for all $n$.

Actually one can assert more: $A \mid F$ is not even closed in $C(F)$. For $F$ contains no perfect subset and $A \mid F$ separates $F$, so that $A \mid F$ is dense in $C(F)$ by [15].

5.3. Our final example relates to 4.6. Let $X=T$, and let $A$ be the subalgebra of $C(X)$ of functions extending continuously to $|z| \leqq 1$ so as to be analytic on $|z|<1, B$ the further subalgebra of those functions whose analytic extensions satisfy $f(0)=f(1)$. Alternatively, an $f$ in $B$ satisfies $f(1)=(1 / 2 \pi) \int_{0}^{2 \pi} f\left(e^{i \theta}\right) d \theta$ $=\lambda(f)$, where $\lambda$ is Haar measure on $T$. Let $\mu_{1}$ be the unit point mass at $1, v=$ $\mu_{1}-\lambda$, so $v \perp B$. As in $4.1, A^{+}+C v$ is weak* dense in $B^{+}$; in fact we can show $A^{+}+C v$ is weak* closed (and so coincides with $B^{\perp}$ ) by the same sort of argument used there. For let $\mu_{\delta} \perp A$, and $\mu_{\delta}+c_{\delta} v \rightarrow \mu$ weak*, where $\left\|\mu_{\delta}+c_{\delta} v\right\| \leqq 1$. Since $v \notin A^{+}$there is an $f$ in $A$ with $v(f)=1$, so $c_{\delta}=\left(\mu_{\delta}+c_{\delta} v\right)(f) \rightarrow \mu(f)=\mathrm{c}$. Consequently $c_{\delta} v \rightarrow c v$ so $\mu_{\delta}=\left(\mu_{\delta}+c_{\delta} v\right)-c_{\delta} v \rightarrow \mu-c v$. And since $\mu_{\delta} \perp A$, $\mu-c v \perp A$, and $\mu \in A^{+}+C v$.

Now let $F=\{1\} \cup\left\{e^{2 \pi i / n}: n=1,2, \cdots\right\}$, and let $\mu \perp B$, so $\mu=\mu^{\prime}+c v$ where $\mu^{\prime} \perp A$. By the F. and M. Riesz theorem $\mu^{\prime}$ is absolutely continuous, so $\mu_{F}=c v_{F}$ $=c \mu_{1}$, and $\left\|\mu_{F}\right\|=|c|$. On the other hand $\mu_{F^{\prime}}=\mu_{F^{\prime}}^{\prime}+c\left(\mu_{1}-\lambda\right)_{F^{\prime}}=\mu^{\prime}-c \lambda$ so $\left\|\mu_{F^{\prime}}\right\|=\left\|\mu^{\prime}-c \lambda\right\|$, which we may write as $\left\|\mu_{F^{\prime}}\right\|=(1 / 2 \pi) \int_{0}^{2 \pi}\left|f\left(e^{i \theta}\right)-c\right| d \theta$ where $f$ is the Radon-Nikodym derivative of $\mu^{\prime}$. Thus

$$
\left\|\mu_{F^{\prime}}\right\| \geqq\left|\frac{1}{2 \pi} \int_{0}^{2 \pi}\left(f\left(e^{i \theta}\right)-c\right) d \theta\right|=\left|\mu^{\prime}(1)-c\right|=|c|
$$

since $\mu^{\prime}(1)=0, \mu^{\prime}$ being in $A^{\perp}$. Consequently we have $\left\|\mu_{F}\right\| \leqq\left\|\mu_{F^{\prime}}\right\|$ for all $\mu$ in $B^{+}$, and by $3.2 B \mid F=C(F)$ is isometrically isomorphic to $B / k F$ : every $f$ in $C(F)$ has an extension $g_{\varepsilon}$ in $B$ with $\left\|g_{\varepsilon}\right\| \leqq(1+\varepsilon)\|f\|$.

But clearly we cannot achieve here the norm preserving extension guaranteed by 4.6 when our algebra is dirichlet; for if $f$ in $C(F)$ is defined by $f(1)=1, f\left(e^{2 \pi i / n}\right)$ $=1-1 / n$ then any $g$ in $B$ with $g \mid F=f,\|g\|=\|f\|=1$, would have an analytic extension $\bar{g}$ to $|z|<1$, bounded by 1 in modulus, with $\bar{g}(0)=\bar{g}(1)=1$, so that $\vec{g} \equiv 1$ on $D$, a contradiction.

Finally, since $B \mid F$ and $B / k F$ are isometric, the remark following 4.13 shows the elements of $F$ are all inequivalent in Gleason's sense, so that 4.13 cannot be improved to assert norm preserving interpolation.

REMARK. An extension of 1.1 should probably be pointed out. Suppose $A$ and $\mathscr{K}$ are as in 1.1, and $B$ is a closed subspace of $C(X)$ which is an $A$ module. Then (a) and (b) of (1.1) hold with $B$ in place of $A$. (2.5 is of course a special case.)

The proof of (a) need only be modified by replacing $A^{+}$by $B^{+}$(as in 2.5). For (b) note that for $\mu \perp B$ and $F$ a peak set of $A$ one has $\mu_{F} \perp B$ by monotone convergence (as in the paragraph preceding 4.13). Since $K \in \mathscr{K}$ is an intersection 
of peak sets one obtains $\mu_{K} \perp B$ by the regularity of $\mu$. Consequently, as in the proof of 3.8, one obtains (3.1) (with $c=1$ ), which implies $B \mid K$ is closed in $C(K)$.

Moreover, since-as we have just observed $-\mu_{F} \perp A$ for $\mu \perp A$ and $F$ any intersection of peak sets, 4.4 extends to any $A$ by 4.8. Indeed if $F=\bigcup_{n=1}^{\infty} F_{n}$ is closed and each $F_{n}$ is an intersection of peak sets, then $\mu_{F} \perp A$ for all $\mu \perp A$ since $\mu_{F_{n}} \perp A$; so by $4.8 F$ is itself an intersection of peak sets if $A$ is separating. Since the reduction to the separating case is obvious, we have: any closed countable union of intersections of peak sets of $A$ is an intersection of peak sets.

(Added in proof.) H. S. Baer has pointed out the following simple proof of this last fact for a pair of peak sets. If $f_{i}$ peaks on $F_{i}, i=1,2$, then $g_{i}=$ $\frac{1}{4}\left(1-f_{i}\right)^{1 / 3} \in A$ since $z \rightarrow(1-z)^{1 / 3}$ is a uniform limit on the spectrum $\sigma_{i}$ of $f_{i}$ of functions analytic near $\sigma_{i}$. From the fact that $-\pi / 6<\arg g_{i}<\pi / 6$ and $\left|g_{i}\right| \leqq \frac{1}{2}$ one easily concludes that $\left|1-g_{1} g_{2}\right| \leqq 1$. Consequently $1-g_{1} g_{2}$ is an element of $A$ peaking on $F_{1} \cup F_{2}$.

\section{REFERENCES}

1. H. S. Bear, Some boundary properties of function algebras, Proc. Amer. Math. Soc. 11 (1960), 1-4.

2. Errett Bishop and Karel deLeeuw, The representation of linear functionals by measures on sets of extreme points, Ann. Inst. Fourier 9 (1959), 305-331.

3. Errett Bishop, A minimal boundary for function algebras, Pacific J. Math. 9 (1959), 629-642.

4. —, Ageneralization of the Stone-Weierstrass theorem, Pacific J. Math.11 (1961), 777-783.

5. —_, A general Rudin-Carleson theorem, Proc. Amer. Math. Soc. 13 (1962), 140-143.

6. Louis deBranges, The Stone-Weierstrass theorem, Proc. Amer. Math. Soc. 10(1959),822-824.

7. A. Browder and J. Wermer, Some algebras of functions on an arc, to appear.

8. Lennart Carleson, Representations of continuous functions, Math. Z. 66 (1957), 447-451.

9. N. Dunford and J. Schwartz, Linear operators. I, Interscience, New York, 1958.

10. K. Hoffman and I. Singer, Maximal algebras of continuous functions, Acta Math. 103 (1960), 217-241.

11. A. M. Gleason, Function algebras, Seminars on Analytic Functions, Vol. 2, pp. 213-226, Institute for Advanced Study, Princeton, N. J., 1957.

12. L. H. Loomis, The spectral characterization of a class of almost periodic functions, Ann. of Math. 72 (1960), 362-368.

13. C. E. Rickart, General theory of Banach algebras, van Nostrand, New York, 1960.

14. Walter Rudin, Boundary values of continuous analytic functions, Proc. Amer. Math. Soc. 7 (1956), 808-811.

15. - Continuous functions on compact spaces without perfect subsets, Proc. Amer. Math. Soc. 8 (1957), 39-42.

16. G. E. Silov, On rings of functions with uniform convergence, Ukrain. Mat. Z. 3 (1951), 404-411, (Russian).

17. John Wermer, Dirichlet algebras, Duke Math. J. 27 (1960), 373-382.

INSTITUTE FOR ADVANCED STUDY,

Princeton, New Jersey

STANFORD UNIVERSITY,

Stanford, California

UNIVERSiTY OF NOTRE DAME,

Notre Dame, Indiana 